\title{
Selective Serotonin Reuptake Inhibitors and Night Sweats in a Primary Care Population
}

\author{
James W. Mold • Barbara J. Holtzclaw
}

Published online: 7 February 2015

(C) The Author(s) 2015. This article is published with open access at Springerlink.com

\begin{abstract}
Objective Several small published case reports have suggested that selective serotonin reuptake inhibitors (SSRIs) can cause night sweats. The purpose of this study was to investigate this possibility further and to explore possible associations between night sweats and other commonly prescribed medications.

Design Cross-sectional, secondary data analysis.

Setting Data were obtained during the Oklahoma Longitudinal Assessment of the Health Outcomes of Mature Adults, a longitudinal cohort study carried out in the Oklahoma Physicians Resource/Research Network.

Participants 413 adult primary care patients aged 65-94 years.

Interventions Current regular use of one of 35 classes of medication.

Main Outcome Measures At least moderate night sweats during the prior month.

Results A total of $38(9.2 \%)$ reported night sweats. Age, gender, body mass index, and total number of medications taken regularly were not associated with night sweats. After adjusting for age and gender, SSRIs (odds ratio [OR] 3.01 ; $95 \%$ confidence interval [CI] 1.26-7.19), angiotensin receptor blockers (ARBs) (OR 3.44; $95 \%$ CI 1.36-8.69), and thyroid hormone supplements (OR 2.53;
\end{abstract}

J. W. Mold ( $\square)$

Department of Family and Preventive Medicine, College of Medicine, University of Oklahoma Health Sciences Center, 900 NE 10th Street, Oklahoma City, OK 73104, USA

e-mail: james-mold@ouhsc.edu

B. J. Holtzclaw

College of Nursing, University of Oklahoma Health Sciences

Center, Oklahoma City, OK, USA
$95 \%$ CI 1.24-5.15) were the only classes of medications associated with night sweats.

Conclusions Use of SSRIs may well be associated with night sweats in older patients. Associations found between night sweats and ARBs and thyroid supplements warrant further study.

\section{Key Points}

The suspected association between selective serotonin reuptake inhibitors (SSRIs) and night sweats is supported.

SSRI effects may be common treatable causes of night sweats in patients seen in primary care.

Associations between thyroid hormone supplements and angiotensin receptor blockers and night sweats deserve further study.

\section{Introduction}

When asked, 10-14\% of seniors and between 34 and $41 \%$ of consecutive adults seen in primary care settings in the USA report experiencing excessive sweating at night $[1,2]$. The estimated annual incidence in adults over 65 years of age is $5 \%[3,4]$. The symptom is rarely reported to clinicians, even by those experiencing "severe" night sweats [1]. In a majority of cases, neither the physician nor the patient has any credible idea about what is causing the symptom [1]. 
Sweating is a complex process controlled primarily, but not entirely, by a thermoregulatory center in the hypothalamus. The eccrine sweat glands are innervated primarily by sympathetic neurons that secrete acetyl choline rather than norepinephrine at their glandular receptor sites. Both sympathomimetic and cholinergic agents can therefore increase sweating. Thermoregulatory sweating is usually accompanied by vasodilatation, which may also contribute to sweat production. Many other factors that can modulate sweating including serum osmolality, circadian rhythm, environmental conditioning, physical fitness, acute and chronic anxiety, sleep disturbances, depression, and hormonal fluctuations (e.g. menopause) [5].

Diaphoresis, in general, is an acknowledged side effect of a number of medications, including non-steroidal antiinflammatory drugs (NSAIDs), narcotics, antiviral agents, fluoroquinolones, angiotensin-converting enzyme inhibitors (ACEIs), angiotensin receptor blockers (ARBs), calcium channel blockers (CCBs), beta adrenergic blockers, vasodilators, anti-estrogenic agents, progesterone, testosterone, thyroid hormone supplements, corticosteroids, insulin, oral hypoglycemic agents, calcitonin, proton pump inhibitors (PPIs), mesalamine, phosphodiesterase inhibitors, tricyclic antidepressants (TCAs), selective serotonin reuptake inhibitors (SSRIs), neuroleptics, cholinesterase inhibitors, anxiolytics, and sedative hypnotics [6]. Sweating is also reported to occur with regular use of inhaled sympathomimetics, inhaled corticosteroids, and some ophthalmologic agents [7-9].

A recently published review of the literature on night sweats found several case studies suggesting that SSRIs can cause night sweats, and that treatment with alpha adrenergic blockers, mirtazapine, cyproheptadine, and/or benztropine might ameliorate them [5, 10-15]. One crosssectional study found an association between night sweats and use of aspirin or acetaminophen [16]. Night sweats also appear to be associated with younger age, menopause, fever, panic attacks, sleep problems, muscle cramps, sensory abnormalities (numbness, impaired vision or hearing), and pain [5]. We retrospectively analyzed data obtained from older patients enrolled in a longitudinal cohort study to see whether we could confirm that regular use of SSRIs was associated with night sweats. We also looked for possible associations between night sweats and 34 other categories of medications.

\section{Methods}

The data used in these analyses were obtained from participants in the Oklahoma Longitudinal Assessment of the Health Outcomes of Mature Adults (OKLAHOMA Studies), a longitudinal cohort study conducted in the
Oklahoma Physicians Resource/Research Network (OKPRN) from 1999 to 2004. The methods used have been published in detail previously [3, 17]. Briefly, 834 individuals aged $\geq 65$ years were initially recruited from the billing record lists of 23 family physicians in central Oklahoma. Patients with known dementia and those living in nursing homes were excluded.

Each participant completed an annual questionnaire that included socio-demographic information, health habits, past and current medical problems, current symptoms, selfrated health, quality of life, and functional status. A research nurse reviewed the questionnaires to ensure that all items were addressed. Participants were re-enrolled annually for up to 4 years, and the questionnaires and examinations were repeated each year at the time of re-enrollment.

The following question about night sweats was included in the questionnaires: "During the last month, how much trouble have you had with night sweats"? Response options included "no trouble, a little trouble, some trouble, a fair amount of trouble, and a great deal of trouble". We converted these responses to a dichotomous variable called "night sweats" (including those reporting some, a fair amount, or a great deal of trouble with night sweats). Only responses made in the third year were considered in this study.

In the third year of the study, participants were asked to bring in all medications they were currently taking regularly (at least twice a week). The medications were recorded and entered into the database within predetermined pharmacologic categories. The category 'analgesics' included NSAIDs, acetaminophen, narcotics, and combinations of narcotics and acetaminophen. We did not include aspirin because, in this cohort, aspirin use was nearly always low dose $(81 \mathrm{mg})$ and presumably not for analgesia. Categories of medications used by fewer than ten patients were excluded from the analyses.

Descriptive statistics were calculated for all variables. Chi squared analyses were used to identify potential associations between "night sweats" and use of any medications within each of the categories (dichotomous). We also examined possible associations between night sweats and potential confounders, including age, gender, body mass index (BMI), and total number of medications taken. We then created separate logistic regression models for each of the medication classes with significant $(p<0.01)$ associations with night sweats in order to adjust for age and gender. BMI and number of medications used were not associated with night sweats or with any medication class and were therefore not included in the regression models. There was an association between use of an SSRI and use of an $\operatorname{ARB}(p=0.01)$, but no associations were found between use of either of these medications and thyroid 
supplements. We therefore included both ARBs and SSRIs in the relevant regression models. Permission was granted by the University of Oklahoma Health Sciences Center Institutional Review Board to conduct these retrospective analyses.

\section{Results}

\section{Study Population}

In year three of the OKLAHOMA Studies, 413 patients aged 65-94 years were enrolled. Their mean (standard deviation [SD]) age was 74.8 (5.5); $231(56 \%)$ were females; $38(9.2 \%)$ reported night sweats. Each of 35 categories of medications was being taken regularly by at least ten participants (see Table 1). The mean (SD) number of different categories of medications taken by participants was 4.9 (2.7). The median and range were 5 and $0-15$.

\section{Associations Between Night Sweats and Medication} Categories

Age, gender, BMI, and number of medications taken regularly were not associated with night sweats. Bivariate associations were found between night sweats and regular use of SSRIs, ARBs, and thyroid hormone supplements but not for any of the other medication categories examined. Adjustment for age and gender did not substantially change these associations. In the adjusted models, patients were again more likely to have reported night sweats if they used SSRIs (odds ratio [OR] 3.01; $95 \%$ confidence interval [CI] 1.26-7.19), ARBs (OR 3.44; $95 \%$ CI 1.36-8.69), and/or thyroid hormone supplements (OR 2.53; $95 \%$ CI 1.24-5.15) (see Table 2).

\section{Discussion}

These findings support the previously reported association between SSRIs and night sweats and raise suspicion that certain other medication classes, particularly ARBs, and thyroid hormone supplements might contribute to night sweats in patients over the age of 65 years.

An estimated $11 \%$ of individuals aged $\geq 12$ years in the USA take antidepressant medications, the majority of whom take SSRIs [18]. Therefore, if SSRIs cause night sweats, they may be one of the more common causes of this symptom in primary care patients. SSRIs might cause night sweats by blocking muscarinic receptors [19] and/or by increasing noradrenergic release, and there is evidence that alpha adrenergic blockers can reduce night sweats in those taking SSRIs [13, 20, 21]. However, it is possible that
Table 1 Number of patients taking medications in each category $(N=413)$

\begin{tabular}{|c|c|}
\hline Medication category & Percentage taking \\
\hline Angiotensin-converting enzyme inhibitors & 97 \\
\hline Alpha blockers & 49 \\
\hline Alpha agonists & 10 \\
\hline Antibacterials & 30 \\
\hline Antineoplastics & 12 \\
\hline Anticholinergics & 46 \\
\hline Cathartics & 49 \\
\hline Anticoagulants & 40 \\
\hline Antihistamines & 85 \\
\hline Anti-Parkinsons & 11 \\
\hline Angiotensin receptor blockers & 32 \\
\hline Aspirin & 179 \\
\hline Benzodiazepines & 29 \\
\hline Other anxiolytics & 12 \\
\hline Beta blockers & 70 \\
\hline Beta agonists & 27 \\
\hline Bisphosphonates & 54 \\
\hline Calcium channel blockers & 78 \\
\hline Corticosteroids & 70 \\
\hline Diuretics & 114 \\
\hline Estrogens & 99 \\
\hline Estrogen/progesterone combinations & 14 \\
\hline Progesterone & 10 \\
\hline $\mathrm{H} 2 \mathrm{~B} / \mathrm{PPIs}$ & 112 \\
\hline Lipid-lowering agents & 139 \\
\hline Insulin & 12 \\
\hline Narcotics & 27 \\
\hline NSAIDs & 133 \\
\hline Oral hypoglycemics & 57 \\
\hline SSRIs & 40 \\
\hline Tricyclic antidepressants & 21 \\
\hline Thyroid & 91 \\
\hline Tylenol & 76 \\
\hline Narcotic/tylenol combinations & 15 \\
\hline Vasodilators & 22 \\
\hline
\end{tabular}

H2B histamine $\mathrm{H} 2$ blockers, NSAID non-steroidal anti-inflammatory drug, $P P I$ protein pump inhibitor, SSRI selective serotonin reuptake inhibitor

it is the underlying depression or anxiety and not the medications that cause nighttime sweating [3]. We were unable to find information in the published literature about how often night sweats occurring in association with SSRIs require discontinuation, reduction in dosage, or treatment with a second medication.

Sweating is listed as a possible side effect of ARBs. The potential mechanism has not been published, to our knowledge, beyond speculation that it may be due to peripheral vasodilatation [22-24]. However, it is not clear 
Table 2 Medication categories associated with night sweats

\begin{tabular}{|c|c|c|c|}
\hline $\begin{array}{l}\text { Medication } \\
\text { category }(N)\end{array}$ & $\begin{array}{l}\text { Night sweats } \\
(N=38)\end{array}$ & OR $(95 \% \mathrm{CI})$ & $p$-value \\
\hline \multicolumn{4}{|c|}{ Selective serotonin reuptake inhibitors } \\
\hline Yes $(40)$ & 9 & $3.01(1.26-7.19)$ & 0.01 \\
\hline No (373) & 29 & & \\
\hline \multicolumn{4}{|c|}{ Angiotensin receptor blockers } \\
\hline Yes (32) & 8 & $3.44(1.36-8.69)$ & 0.009 \\
\hline No (379) & 30 & & \\
\hline \multicolumn{4}{|c|}{ Thyroid hormone supplement } \\
\hline Yes (91) & 15 & $2.53(1.24-5.15)$ & 0.007 \\
\hline No $(320)$ & 23 & & \\
\hline
\end{tabular}

$C I$ confidence interval, $O R$ odds ratio

why this mechanism should induce sweating only at night. In speculating about possible mechanisms, it is worth considering which patients are more likely to take ARBs. For example, ARBs are more likely to be used by individuals who were unable to tolerate ACEIs because of cough or angioedema. Perhaps the metabolic idiosyncrasies associated with those side effects are related to sweating at night.

Thyroid hormone replacement or supplements can produce sweating if given in excess, producing a hyperthyroid state of increased thermogenesis, systemic catecholamine levels, and/or catecholamine receptor hypersensitivity [2528]. Inadvertent overtreatment with synthetic thyroxin, in particular, might induce sweating. Overtreatment is often due to reliance on the serum thyroid-stimulating hormone (TSH) level, which may or may not be a true reflection of thyroid hormone activity in tissues, especially in the elderly [29].

The study has several important limitations. First, the number and proportion of participants reporting night sweats was small, and the few participants within some of the medication categories reduced our power to detect associations with night sweats and those medication classes. Second, it was a cross-sectional study, precluding us from drawing firm conclusions about causation. Further, some potential confounders like depression were not considered, though there is no reason to believe that the predictors of night sweats identified in prior studies (e.g. fever, muscle cramps, and subjective sensory impairments) are associated with use of SSRIs, ARBs, or thyroid hormones.

Finally, sweating and symptomatic complaints related to sweating tend to diminish with increasing age. This response was tested, with thermal stimulation and intradermal acetylcholine or methacholine, in 46 men and women aged $>70$ years compared with younger adults [30]. A higher threshold for sweating was found in most of the older adults compared with the younger controls, with an even higher threshold for older women than men. These findings suggest that a cohort of older people may not be the best population sample in which to study the association of medications with sweating. In addition to possibly under-representing the sweat-inducing potential of drugs, older people are more likely to have more medical conditions and take more medications, increasing the unmeasured variability in our analyses. However, the fact remains that prior studies have reported night sweats but failed to identify or investigate associations between their incidence and possible drugs that cause them.

\section{Conclusions}

The findings of this study are consistent with other published evidence that SSRIs are associated with and may cause night sweats. Because of their frequency of use, this may be one of the more common causes of night sweats in patients seen in primary care. The other medication classes implicated in this study, ARBs and thyroid hormone supplements, deserve further study.

Disclaimer The views expressed in the submitted article are our own and not an official position of the institution or funder.

Source of support Presbyterian Health Foundation.

Conflict of interest None declared.

Open Access This article is distributed under the terms of the Creative Commons Attribution Noncommercial License which permits any noncommercial use, distribution, and reproduction in any medium, provided the original author(s) and the source are credited.

\section{References}

1. Mold JW, Mathew MK, Belgore S, DeHaven M. Prevalence of night sweats in primary care patients: an OKPRN and TAFP-Net collaborative study. J Fam Pract. 2002;51(5):452-6.

2. Mold JW, Woolley JH, Nagykaldi Z. Associations between night sweats and other sleep disturbances: an OKPRN study. Ann Fam Med. 2006;4(5):423-6.

3. Mold JW, Roberts M, Aboshady HM. Prevalence and predictors of night sweats, day sweats, and hot flashes in older primary care patients: an OKPRN study. Ann Fam Med. 2004;2(5):391-7.

4. Mold JW, Lawler F. The prognostic implications of night sweats in two cohorts of older patients. J Am Board Fam Med. 2010;23(1):97-103.

5. Mold JW, Holtzclaw BJ, McCarthy L. Night sweats: a systematic review of the literature. J Am Board of Fam Med. 2012;25(6): 878-93.

6. Cheshire WP, Fealey RD. Drug-induced hyperhidrosis and hypohidrosis: incidence, prevention and management. Drug Saf. 2008;31(2):109-26.

7. Lemp MA. Management of dry eye disease. Am J Manag Care. 2008;14(3 Suppl):S88-101. 
8. Foster JM, Aucott L, van der Werf RH, et al. Higher patient perceived side effects related to higher daily doses of inhaled corticosteroids in the community: a cross-sectional analysis. Respir Med. 2006;100(8):1318-36.

9. Primatene Side Effects Center. RxList Inc. 2014. http://www. rxlist.com/primatene-side-effects-drug-center.htm. Accessed 17 April 2014.

10. Pierre JM, Guze BH. Benztropine for venlafaxine-induced night sweats. J Clin Psychopharmacol. 2000;20(2):269.

11. Buecking A, Vandeleur CL, Khazaal Y, Zullino DF. Mirtazapine in drug-induced excessive sweating. Eur J Clin Pharmacol. 2005;61(7):543-4.

12. Mago R, et al. Antidepressant-induced excessive sweating (ADIES): measurement and treatment. Paper presented at: 162nd Annual Meeting. Shaping our Future: Science and Service; May 16-21, 2009; San Francisco.

13. Mago R, Monti D. Antiadrenergic treatment of antidepressantinduced excessive sweating in 3 patients. J Clin Psychiatry. 2007;68(4):639-40.

14. Ahmed A. Sertraline-related night sweats. Am J Geriatr Psychiatry. 2002;10(4):484.

15. Ashton AK, Weinstein WL. Cyproheptadine for drug-induced sweating. Am J Psychiatry. 2002;159(5):874-5.

16. Lea MJ, Aber RC. Descriptive epidemiology of night sweats upon admission to a university hospital. South Med J. 1985;78(9): 1065-7.

17. Mold JW, Vesely SK, Keyl BA, Schenk JB, Roberts M. The prevalence, predictors, and consequences of peripheral sensory neuropathy in older patients. J Am Board Fam Pract. 2004;17(5): 309-18.

18. Pratt LA, Brody DJ, Gu Q. Antidepressant use in persons aged 12 and over: United States, 2005-2008. NCHS Data Brief. 2011;76:1-8.
19. Anderson I, Nutt D, Deakin J. Evidence-based guidelines for treating depressive disorders with antidepressants: a revision of the 1993 British Association for Psychopharmacology guidelines. J Psychopharmacol. 2000;14(1):3-20.

20. Stahl SM, Grady MM, Moret C, Briley M. SNRIs: their pharmacology, clinical efficacy, and tolerability in comparison with other classes of antidepressants. CNS Spectr. 2005;10(9):732-47.

21. Marcy TR, Britton ML. Antidepressant-induced sweating. Ann Pharmacother. 2005;39(4):748-52.

22. Warnholtz A, Ostad MA, Heitzer T, et al. AT1-receptor blockade with irbesartan improves peripheral but not coronary endothelial dysfunction in patients with stable coronary artery disease. Atherosclerosis. 2007;194(2):439-45.

23. Mirza FS, Ong P, Collins P, et al. Effects of estradiol and the angiotensin II receptor blocker irbesartan on vascular function in postmenopausal women. Menopause. 2008;15(1):44-50.

24. Koifman B, Topilski I, Megidish R, et al. Effects of losartan + Larginine on nitric oxide production, endothelial cell function, and hemodynamic variables in patients with heart failure secondary to coronary heart disease. Am J Cardiol. 2006;98(2):172-7.

25. Silva JE. Thyroid hormone and the energetic cost of keeping body temperature. Biosci Rep. 2005;25(3-4):129-48.

26. Robertshaw D. Hyperthidrosis and the sympatho-adrenal system. Med Hypotheses. 1979;5(3):317-22.

27. Matsumoto T, Kosaka M, Yamauchi M, et al. Effect of thyroid hormone on thermal sweating. Trop Med. 1988;30(3):225-31.

28. Waldstein SS. Thyroid-catecholamine interrelations. Annu Rev Med. 1966;17(1):123-32.

29. Al-Adsani H, Hoffer LJ, Silva JE. Resting energy expenditure is sensitive to small dose changes in patients on chronic thyroid hormone replacement. J Clin Endocrinol Metab. 1997;82(4):1118-25.

30. Foster KG, Ellis FP, Dore C, Exton-Smith AN, Weiner JS. Sweat responses in the aged. Age Ageing. 1976;5(2):91-101. 